\title{
THE EFFECT OF COMPRESSED Air Massage on Untraumatised Rabbit SKeletal Muscle - A Morphometric AND ULTRASTRUCTURAL STUDY
}

\begin{abstract}
One of the therapeutic uses of massage is to facilitate repair of damaged tissue. A potential hazard of massage is that direct pressure on muscle is known to damage skeletal myofibres. This study examines the effect of a new form of massage using compressed air on the morphology of untraumatised rabbit skeletal myofibres.

Under anaesthetic, the left and right vastus lateralis muscles of 16 New Zealand, white rabbits were treated with 10 minutes of compressed air therapy at 1 Bar using a single hole $(5 \mathrm{~mm})$ applicator head and control

GREGORY MA, PhD'; MARS M, MBChB, MD²

${ }^{1}$ Emeritus Professor, Electron Microscopy Unit, University of KwaZulu-Natal.

2 Professor and Head of TeleHealth, Nelson R. Mandela School of Medicine, University of KwaZulu-Natal, Durban, South Africa.

biopsies were taken from the opposite limb. Biopsies were prepared for light microscopy and transmission electron microscopy. Morphometry, using image analysis revealed a significant increase in myofibre diameters 10 minutes $(p<0.001)$ and 24 hours $(p<0.01)$ after compressed air massage. Six days after treatment diameters were significantly reduced $(p<0.01)$. Morphologically, myofibres in control specimens were normal. Shortly after compressed air massage, juxta-nuclear and intermyofibrillar oedema was present and electron-lucent spaces were filled with swollen mitochondria and elements of the sarcoplasmic reticulum (SR). Glycogen and other non-contractile organelles were sometimes aggregated in oedematous, sub-sarcolemmal regions. Twenty four hours after treatment, intermyofibrillar oedema was reduced, but SR swelling remained and many fibres were characterised by focal and large areas of myofibrillar disorganisation. With the exception of occasional swollen elements of the SR and a single internalised nucleus, myofibres morphology had returned to normal 6 days after treatment. Compressed air massage causes less damage to skeletal myofibres than a similar form of localised pressure treatment, deep transverse frictions. Clinical studies using this new modality are warranted.
\end{abstract}

KEY WORDS: COMPRESSED AIR MASSAGE, SKELETAL MUSCLE, ULTRASTRUCTURE, MORPHOMETRY, ELECTRON MICROSCOPY.

\section{INTRODUCTION}

Massage is perhaps the oldest of the healing crafts, with surviving documents from China and India mentioning "rubbing" as a form of therapy up to 5000 years ago. Hippocrates (circa 430 BC), is credited with saying, "a little rubbing is beneficial" (Kaments 1960). More recently, in 1847, Per Henrik Ling developed and published a comprehensive system of massage, which today is known as "Swedish Massage Therapy", a modality that covers a wide range of manual manipulations of the human body (effleurage, petrissage, frictions, stroking and vibration) (Beard and Wood 1965).

Massage is reported to impart a broad spectrum of benefits on a wide range of muscular-skeletal injuries and medical and psychological conditions (Field 1998, Goats 1994). It is widely used to improve and hasten healing of soft-tissue injuries (Smith et al 1994), to improve lymph drainage in lymphoedema (Casley-Smith et al 1998, Fiaschi et al 1998, Kurz et al 1981) and generally improve blood circulation in oedematous limbs (Goats 1994). Massage has also proved beneficial in the management of chronic pain associated with fibromyalgia (Sunshine et al 1996), rheumatoid arthritis, (Field et al 1997a) acute pain experienced during childbirth (Waza et al 1993) and after the debridement of burns (Field 1998). It is reported to prevent adherent scar formation after surgery (Norris 1993) and it has also been used to reduce the symptoms of such conditions as asthma, (Field et al 1998) diabetes (Field et al 1997b) and enhance the immune systems' cytotoxic capacity (Diego et al 2001, Ironson et al
1996). In addition, massage has also been used to reduce stress (Cady and Jones 1997).

A key aim of massage in the treatment of soft tissue lesions is to facilitate repair and the return to normal tensile strength of the damaged tissue, with the production of a flexible functional scar (Hunter 1994).

\section{CORRESPONDENCE TO:}

Professor Maurice Mars

Department of Physiology

Nelson R Mandela School of Medicine

University of KwaZulu-Natal

Private Bag 7, Congella 4013

South Africa

Tel: $\quad$ +27312604364

Fax: $\quad+27312604455$

E-mail:mars@nu.ac.za 
A new form of treatment has recently been developed, that employs compressed air as a massage modality (Jet Therapy®). When compressed air is applied through a $5 \mathrm{~mm}$ diameter hole at pressures of one to two Bar, the pressures generated in skeletal muscle are high (Mars 2002) and the effect may be similar to that of deep transverse friction (DTF). Deep transverse friction provides therapeutic movement over a small area and allows pressure to be applied to greater depth in muscle than effleurage or petrissage modalities. It has been used for the treatment of muscle strains and tears, tenosynovitis, tendinitis and ligament strains (Fritschy and de Gautard 1988, Gimblett et al 1999, Hammer 1993). A potential hazard of massage, especially a technique such as DTF is that direct and indirect pressure on muscle may damage skeletal myofibres (Mars and Hadley 1998). DTF has recently been shown to alter the morphology of underlying myofibres, producing a time dependent cascade of reversible changes that include myofibre oedema, supercontraction of myofibres, two types of inclusions and other sarcoplasmic alterations (Deane et al 2002, Gregory et al 2002, Gregory et al 2003).

The aim of this study was to examine the effect of compressed air massage at 1 Bar on the morphology of untraumatised rabbit skeletal myofibres and to compare this with reported data on the changes in rabbit skeletal myofibres induced by DTF.

\section{METHODS}

Sixteen New Zealand, white rabbits were studied with the approval of the Ethics and Research Committees of the University of Natal and the University of Durban-Westville. The animals were housed in the Biomedical Resource Centre (BRC) of the University of Durban Westville and were maintained under the care of the staff of the BRC. The animals were fed ad libitum. Before compressed air massage, anaesthesia and analgesia were achieved by an intramuscular injection of a combination of $50 \%$ Ketamine and $50 \%$ Xylazine, $10 \mathrm{mg} / \mathrm{kg}$ of rabbit body weight. After treatment, the animals were observed during recovery from the anaesthetic and then returned to the holding facility. Subsequent muscle biopsy was performed under anaesthetic and the animal euthenased.

Figure 1: Compressed air massage unit consisting of two reservoir tanks, a regulator valve and inflow and outflow tubing.

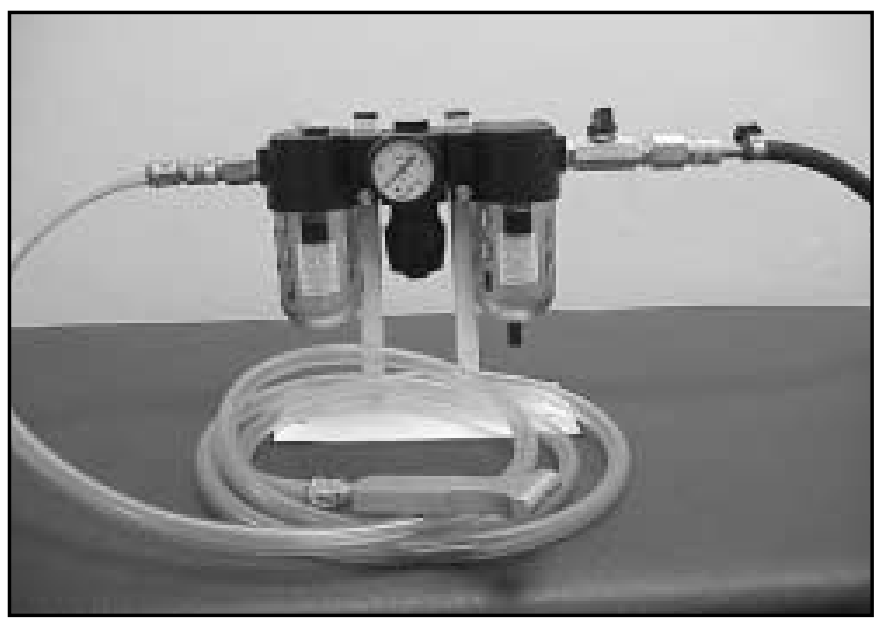

\section{Compressed Air Apparatus and Treatment}

The apparatus consists of an electrically driven air compressor, two reservoir tanks, medical air filters, a pressure regulator valve, pressure tubing and several different applicator heads (Figs. 1 and 2). Compressed air at pressures of 4 to $7 \mathrm{Bar}$ passes from the compressor into the first reservoir tank, where the air is filtered to remove any particulate matter. A regulator valve controls the pressure at which compressed air is released from the first reservoir tank into the second reservoir tank and subsequently through compression hosing to an applicator head. Treatment involves gently massaging skin with the metal applicator head and the stream of compressed air that passes through the applicator head supplements the massage. The applicator heads differ in the number, size and position of the holes through which the air passes. Air pressures for treatment range from 1 to 2 Bar, and additional pressure may be applied to the skin by the therapist pushing the applicator head down on the skin during the massage.

An applicator head with a single $5 \mathrm{~mm}$ diameter hole was used in this study and compressed air was applied at a pressure of 1 Bar for 10 minutes. This applicator head was selected as it produces the highest localized pressure on subcutaneous tissue and muscle (Mars 2002). The left vastus lateralis was treated at the level of the mid-thigh. The fur was removed with a depilatory, prior to treatment, to enable observation of any inflammatory reaction and to facilitate treatment. During treatment the applicator head and compressed air jet was moved proximally and distally over the lateral thigh.

\section{Muscle Biopsy and Preparation}

The skin was incised longitudinally over the treatment site, the fascia opened and a wedge biopsy of approximately $1 \mathrm{~cm}^{3}$ of the mid portion of the vastus lateralis muscle excised. Samples were taken from the left vastus lateralis within 10 minutes (RE1 - RE4), 24 hours (RE5 - RE8) and 6 days after treatment (RE9 - RE12). Similar biopsies were taken from the right vastus lateralis of each animal ( $\mathrm{RC} 1$ - $\mathrm{RC} 12)$, to serve as controls. To confirm that the vastus lateralis muscle in one leg could serve as a control for the same muscle in the other leg, biopsies were

\section{Figure 2: Compressed air applicator heads. Configura- tion ranges from a single large diameter $5 \mathrm{~mm}$ hole to a long narrow slit.}

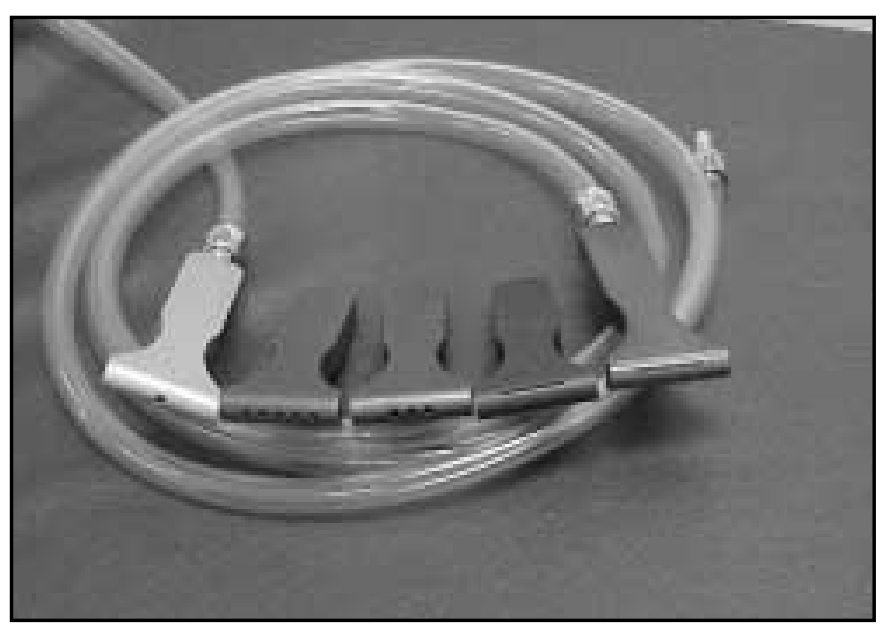


taken from the left and right vastus laterali of an additional 4 untreated animals (CL13 - CL16: CR13 - CR16). To reduce biopsy induced, muscle "supercontraction" artefact, the entire biopsy was immediately immersed in Karnovsky's fixative (Karnovsky 1965) as per the method of Olmesdahl (Olmesdahl et al 1979). After 5 minutes immersion, the biopsy was examined with a stereomicroscope to determine the alignment of myofibres. Each biopsy was bisected, one being prepared for light microscopic morphometry, the other for transmission electron microscopy (TEM).

\section{Light Microscopic Morphometry}

The tissue was re-immersed in Karnovsky's fluid for 24 hours prior to dehydration and perfusion and embedding in wax. Care was taken to orientate the tissue to facilitate the crosssectioning of myofibres. Sections of $3 \mu \mathrm{m}$ were cut of the wax embedded tissue, mounted on glass slides and stained with haematoxylin and eosin ( $\mathrm{H}$ and $\mathrm{E}$ ). The sections were examined using a light microscope with a $10 \mathrm{X}$ objective and the crosssectional diameter of myofibres measured using an image analyser and SIS software. The morphometric method employed was that described by Mars (Mars and Gregory). In brief, suitable areas containing transverse/obliquely sectioned myofibres were selected for morphometric evaluation. The myofibres were considered to be near cylinders and the diameter of fibres taken to be the minimum distance across each cell. A minimum of 100 fibres were measured from each control and experimental specimen. The mean, standard deviation and error of the mean was determined for the fibres within each animal and for each group of animals. Comparisons of means within and between groups was by analysis of variance using the Kruskal-Wallace test with post-hoc testing using Dunn's multiple comparison test. A nonparametric test was chosen because Bartlett's test revealed significant differences in the standard deviations of the groups. Significance levels were set at, $\mathrm{p}<0.05$.

\section{Transmission Electron Microscopy (TEM)}

Tissue for TEM was minced into blocks approximately $0.5 \mathrm{~mm}$ by $1 \mathrm{~mm}$ in such a manner as to ensure that myofibres were oriented longitudinally through the long axis of each block. The blocks were re-immersed in fresh Karnovsky's fixative for 1 hour, secondarily fixed in $1 \% \mathrm{OsO}_{4}$, block stained in $1 \%$ aqueous uranyl acetate, dehydrated through graded ethanols and propylene oxide and perfused with Spurr's epoxy resin (Spurr 1969). The blocks were embedded in silicone moulds and orientated to facilitate either the transverse or longitudinal sectioning of myofibres. Survey sections, $1 \mu \mathrm{m}$ in thickness of both longitudinal and transversely orientated myofibres were stained with $1 \%$ aqueous, alkaline toluidine blue, examined by light microscopy, and areas of interest selected for ultrastructural evaluation. Ultrathin sections, approximately $60 \mathrm{~nm}$ in thickness were cut using diamond knives, picked up on uncoated, 200 mesh copper grids and double stained with Reynolds' lead citrate (Reynolds 1963) and ethanolic uranyl acetate. The sections were examined with a Jeol 1010 transmission electron microscope at $60 \mathrm{kv}$.

\section{RESULTS}

All animals developed ecchymosis of the skin during compressed air massage which was present for up to 24 hours after treatment. There was minimal or no hyperaemia immediately after treatment with little bleeding in biopsies taken within 10 minutes of massage. Maximal hyperaemia was present 24 hours after treatment and 5 days later minimal or no hyperaemia was noted in any animal. The air pressure was sufficient to tear the skin of animal 7. This resulted in surgical emphysema, which was still present 24 hours later at biopsy.

\section{Morphometry}

Most myofibres in all specimens were cut in cross-section and appeared as irregular polygons grouped into bundles. Mean diameters of fibres (FD) in the 4 specimens of the untreated left and right vastus lateralis were $36.6 \pm 10.6 \mu \mathrm{m}(95 \% \mathrm{CI}$, 35.92 - 36.64) and $35.7 \pm 10.4 \mu \mathrm{m}$ (95\% CI, $35.01-36.37$ ) respectively. No statistical differences were detected between either the marginal distribution of FD or FD group means.

The mean morphometric FD data obtained from control and massaged muscle $10 \mathrm{~min}, 24 \mathrm{hr}$ and 6 days after treatment are shown in figure 3 . Within $10 \mathrm{~min}$ of massage, average increase in FD was $17.2 \%$ ( $\mathrm{p}<0.001)$. Twenty four hours after massage, the average increase was $5.5 \%(p<0.002)$. Six days after treatment, the average fibre diameter of the treated muscle was reduced by $5 \%(\mathrm{p}<0.05)$.

The marginal distribution of fibres is shown in Figure 4. The control distribution is derived from FD obtained from all untreated specimens $(n=3804)$. Note that in control specimens, there is a near normal distribution of FD with the mode (36\%) at $35 \mu \mathrm{m}$. Within 10 minutes of massage, the distribution is significantly skewed to larger FD dimensions with the mode (26\%) at $45 \mu \mathrm{m}$. Twenty four hours after massage, while the distribution is near normal, the mode (37\%) still at $45 \mu \mathrm{m}$. Six days after treatment, the distribution almost mirrors that of control specimens the mode (34\%) returning to $35 \mu \mathrm{m}$.
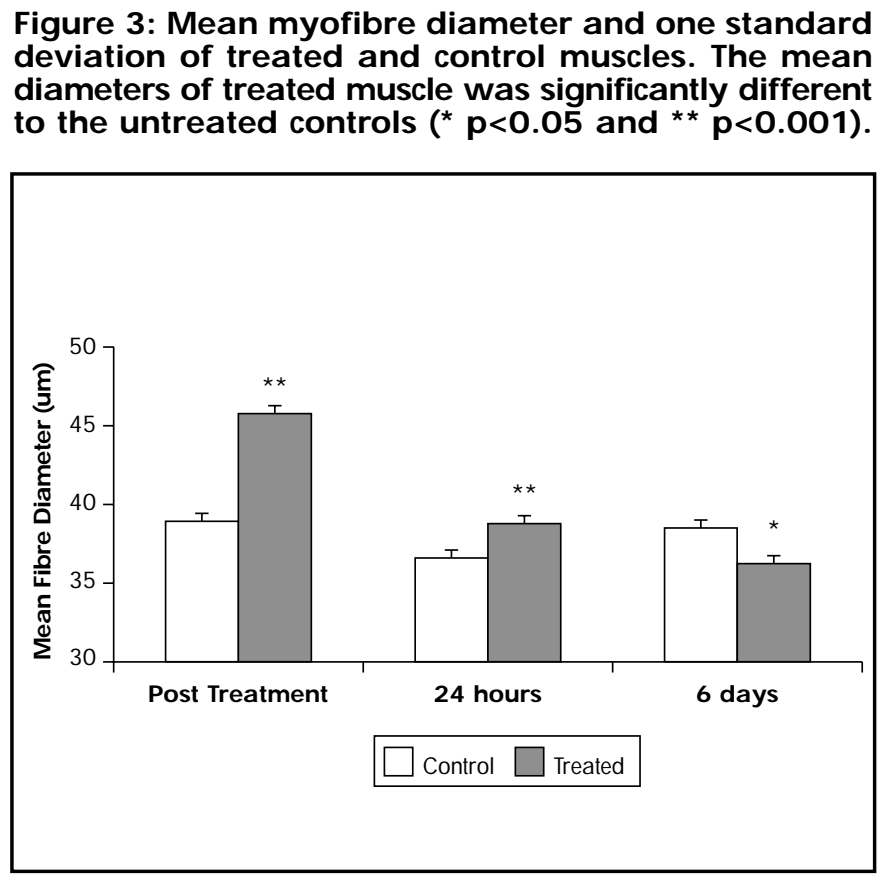
Figure 4: Frequency polygons showing the distribution of fibres of a particular diameter 10 minutes, 24 hours and 6 days after compressed air massage. The control distribution of fibres in all control specimens is plotted for comparison.

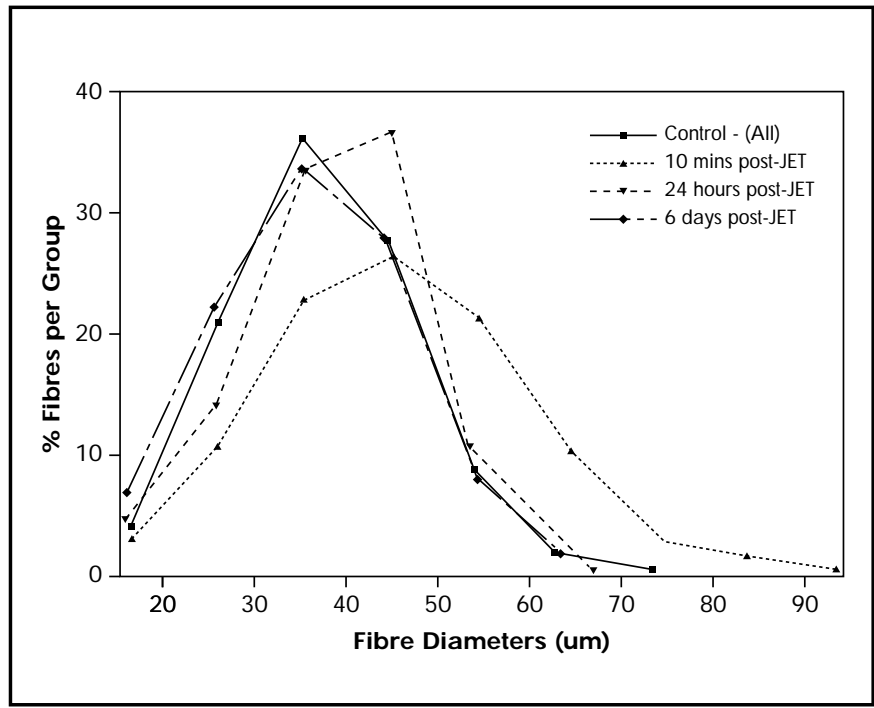

\section{Transmission Electron Microscopy}

Control: The myofibres in control tissue from right leg vastus lateralis muscles were similar to the ultrastructural descriptions of normal mammalian skeletal (Dubowitz 1985). Further, they were similar in every respect to the recent ultrastructural descriptions by this group of normal rabbit biceps femoris muscle (Gregory et al 2003). In longitudinal section, nuclei were situated beneath the sarcolemma and basal lamina and appeared as elongated ovals up to $25 \mu \mathrm{m}$ in length and $10 \mu \mathrm{m}$ in diameter. While prominent nucleoli were present in some nuclei, chromatin was generally distributed in small aggregates throughout the nucleosol, especially beneath the nucleolemma. Myofibrils were composed of sarcomeres from $1.7 \mu \mathrm{m}$ to $2 \mu \mathrm{m}$ in length, with Z-band "synchrony" generally present over the full width of each fibre. Mitochondria in the juxta-nuclear and intermyofibrillar spaces averaged $0.3 \mu \mathrm{m}$ in diameter and up to $2 \mu \mathrm{m}$ in length and contained densely packed cristae within a

Figure 5: Control - Myofibrils (M) and mitochondria $(m)$ in an apparenty normal myofibre. t-tubes (arrow ed)

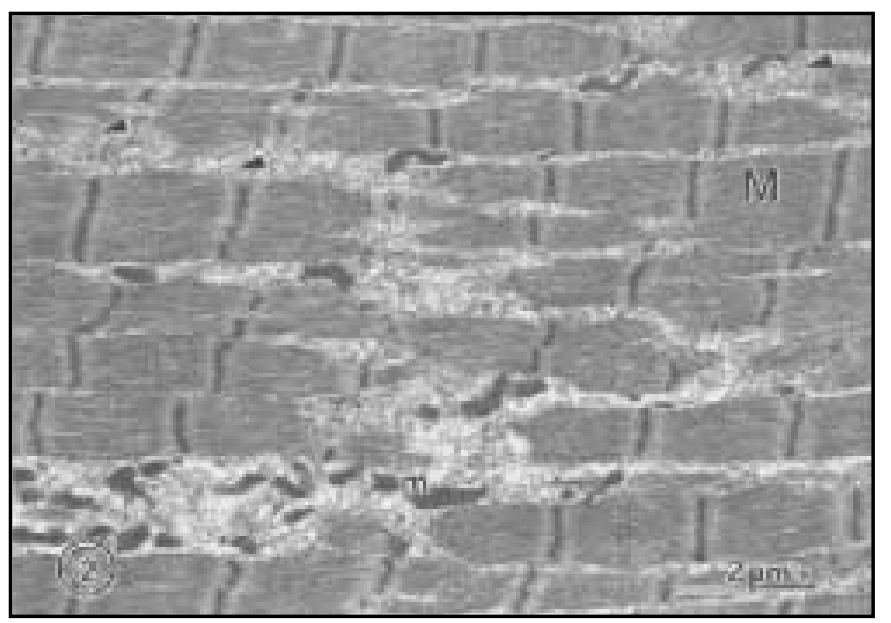

moderately electron-dense mitochondriosol. T-tubes were not swollen and the intermyofibrillar space contained substantial quantities of $\alpha$-glycogen. Sarcoplasmic reticulum and Golgi cisternae appeared normal (Figure 5). Supercontraction bands were not present in any fibre suggesting that the preventative measures taken to preclude preparation mediated mechanical artefact had been successful.

\section{Within 10 minutes of compressed air massage:}

While most nuclei appeared ultrastructurally normal, many juxta-nuclear regions contained swollen mitochondria and other sarcoplasmic organelles (Figure 6). Intermyofibrillar spaces were wider than in control tissue and contained swollen elements of the sarcoplasmic reticulum. Such regions usually contained apparently normal t-tubes together with normal and swollen mitochondria within a glycogen rich matrix (Figure 7). Other fibres had wide, oedematous intermyofibrillar spaces

Figure 6: <10 minutes post-therapy - Swollen (sm) and normal mitochondria (sm) and swollen elements of the smooth endoplasmic reticulum (s) near a normal nucleus $(\mathrm{N})$. b = basal lamina; sarcolemma (arrow ed).

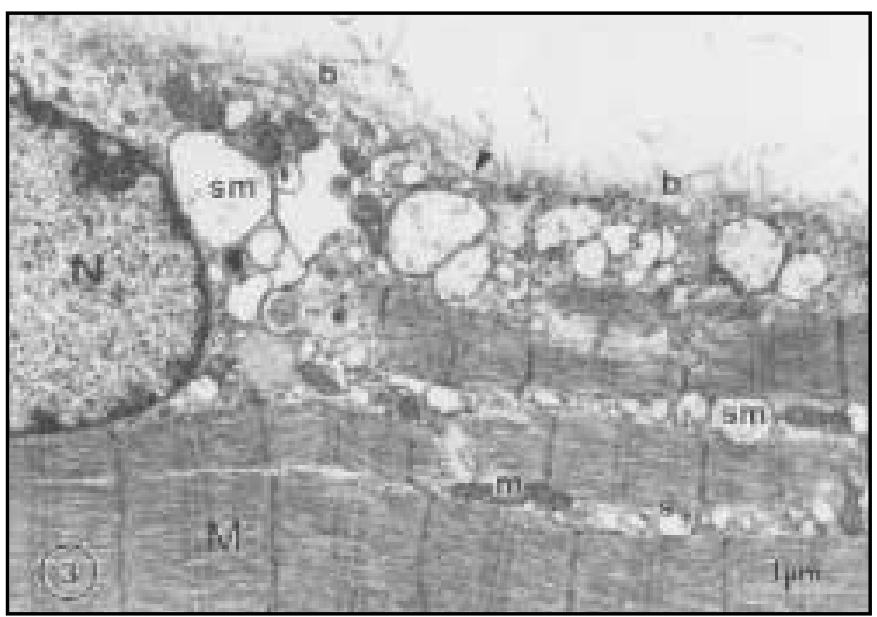

Figure 7: <10 minutes post-therapy - Separated myofibrils with a sarcosol rich in glycogen (g). Note numerous sw ollen elements of the SER, sw ollen mitochondria and disorganised myofilaments (f). Normal t-tubes (arrowed)

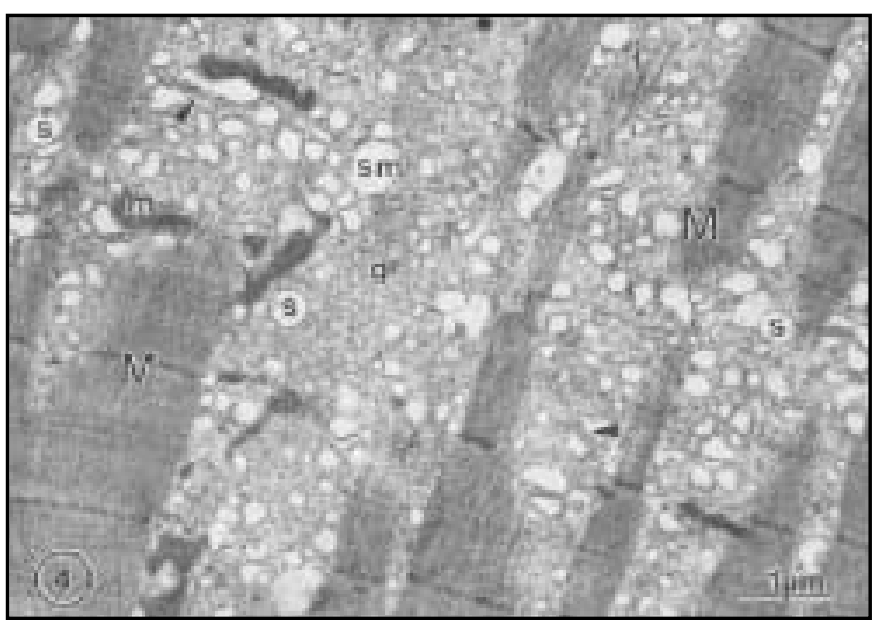


Figure 8: <10 minutes post-therapy - Oedematous region ( 0$)$ between myofibrils. Note apparent sarcomere disorganisation (f). Normal t-tubes (arrowed).

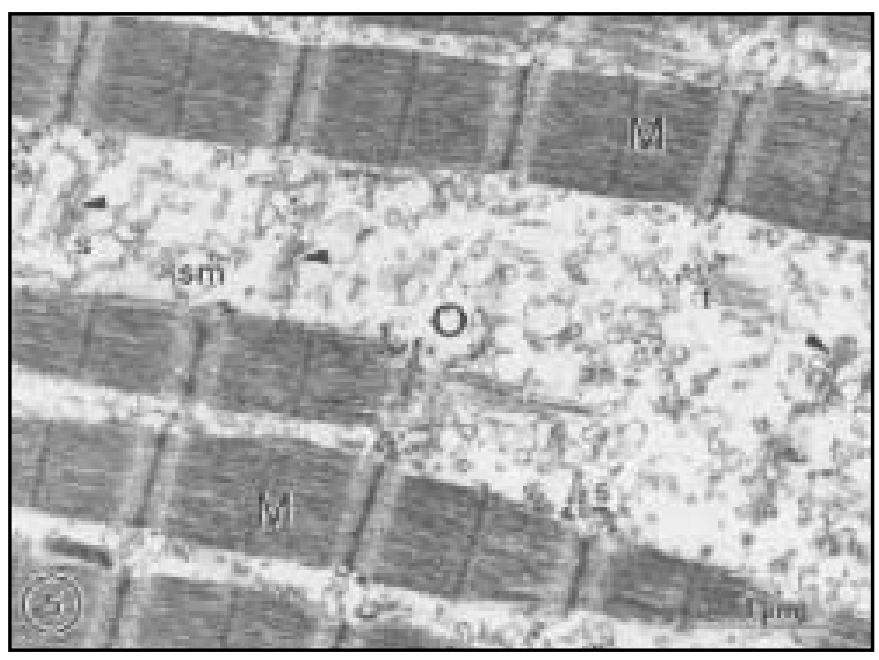

Figure 9: <10 minutes post-therapy - Stretched (ss) and contracted sarcomeres (cs) near the periphery of a fibre.

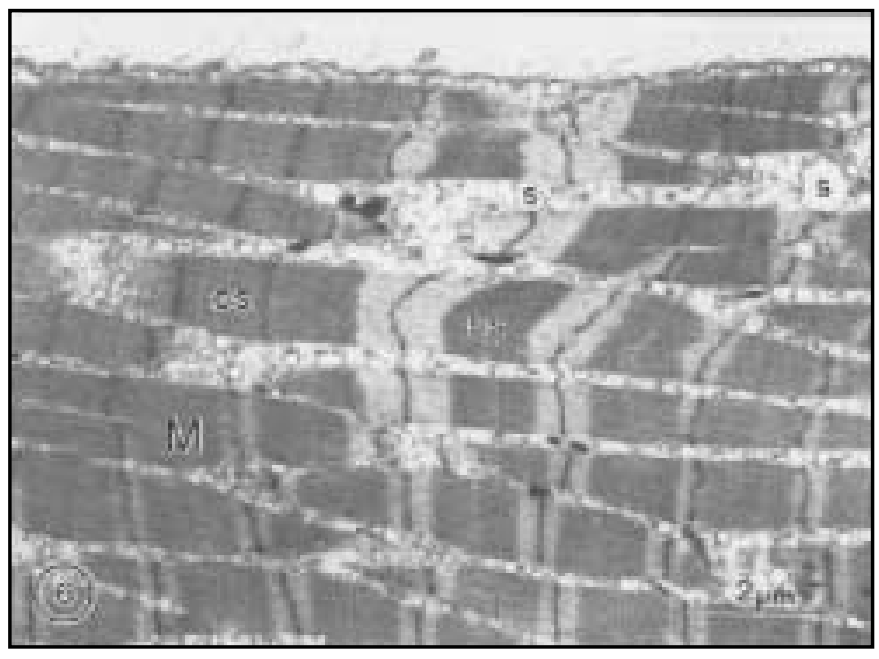

Figure 10: <10 minutes post-therapy - Oedematous subsarcolemmal "sac" (O) containing aggregates of glycogen (g) and occasional small mitochondria. Basal lamina (arrowed).

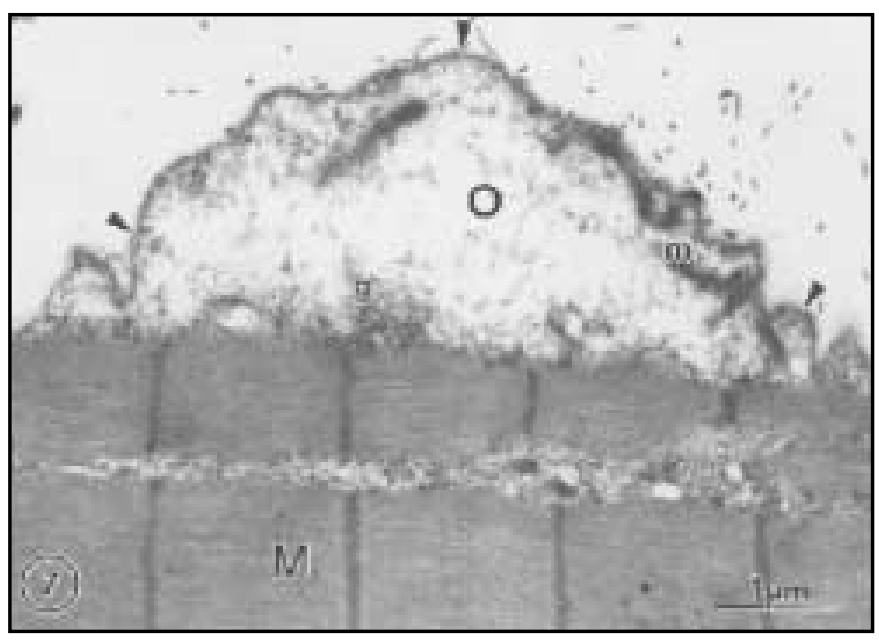

devoid of glycogen and other particulate material within which were numerous membrane-bound vacuoles. The t-system in such areas was difficult to identify, but when located was not swollen (Figure 8). While there were no supercontraction bands in any fibre, there were some myofibrillar irregularities. In oedematous fibres, there was focal disorganization of individual and small groups of sarcomeres with actin and myosin filaments in disarray (Figures $7 \& 8$ ). Usually in less swollen fibres, peripheral myofibrils were "stretched" on the periphery of fibres, thereby causing asynchrony of Z-bands across the fibre. Z-band asynchrony was exacerbated by the stretching $(3.2 \mu \mathrm{m})$ and contraction $(1.5 \mu \mathrm{m})$ of sarcomeres within individual myofibrils (Figure 9). Of particular note were the large, oedematous subsarcolemmal spaces, some exclusively filled with glycogen, some with glycogen together with occasional mitochondria and others empty save for occasional mitochondria and other small sarcoplasmic organelles (Figure 10).

\section{4 hours after compressed air massage:}

Numerous vacuoles, presumably dilated elements of the sarcoplasmic reticulum, were present in widened intermyofibrillar spaces in most fibres. Some vacuoles were embedded in a matrix rich in glycogen (Figure 11), while others were located in a particularly electron-lucent sarcosol similar to that shown in Figure 8. Irrespective of the matrix, the mitochondria and t-system appeared normal. Many myofibres had regions where the integrity of sarcomeres had broken down. In some fibres, the dissolution of sarcomeres was restricted to 3 or 4 sarcomeres in a single myofibril while in others, sarcomere dissolution extended over a significant proportion of the fibre (Figure 12). While focal groups of contracted sarcomeres caused myofibrillar, Z-band asynchrony in some fibres, only one example of supercontraction was found in one fibre (Figure 13). The contraction band extended across most of the fibre and was comprised of 18 sarcomeres with an average length of $0.4 \mu \mathrm{m}$. Adjacent sarcomeres were "stretched" up to $3.3 \mu \mathrm{m}$ in length. The nucleus of this fibre was crenated and the nucleolemma swollen. Other than this single aberration,

Figure 11: 24 hours post-therapy - Normal mitochondria and myofibrils with a sarcosol rich in glycogen and replete with swollen SER (s).

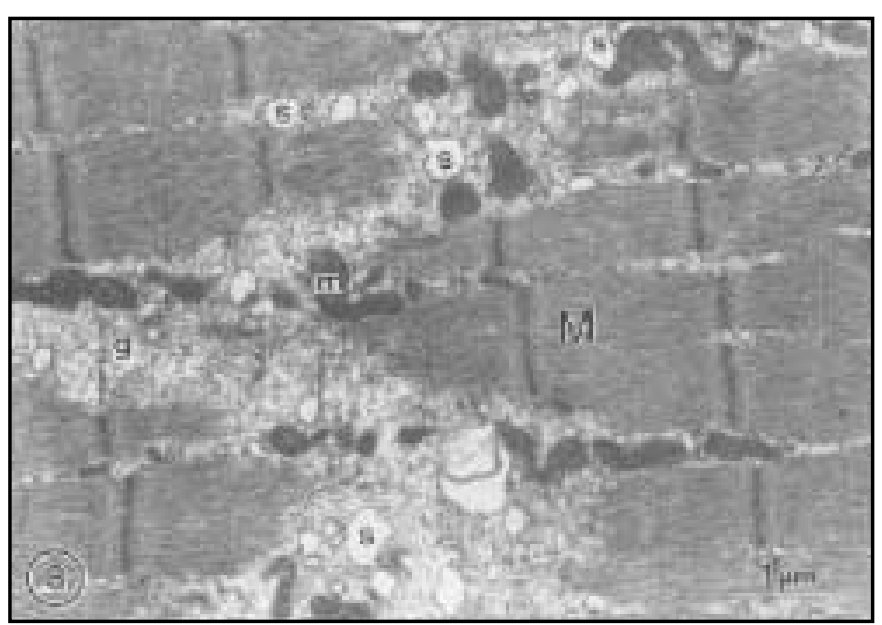


and a very occasional, morphologically normal internalised nucleus, the general appearance of nuclei in this group of specimens was normal.

Treatment ruptured the skin of animal 7 and occasional groups of erythrocytes were seen in the interfibre spaces. Other than this, the morphological appearance of myofibres in this animal was similar to those in muscle from other animals in this group.

\section{6 days after compressed air massage:}

Most fibres appeared morphologically normal with no evidence of oedema in either the sarcoplasm or t-system (Figure 14). There were, however, occasional fibres where the sarcoplasmic reticulum remained dilated and where intermyofibrillar vacuoles were still present (Figure 15). While still exhibiting some abnormalities, such fibres contained apparently normal mitochondria, nuclei and other non-reticular, sarcoplasmic organelles. Glycogen levels were normal and there was no evidence of t-tube swelling.

\section{DISCUSSION}

Compressed air therapy (Jet Therapy®) is a novel massage technique that focuses a stream of compressed air on skin and subcutaneous tissue. At applied pressures of 1 - 2 Bar, compressed air therapy falls into the category of a deep effleurage or friction massage technique and could be used as an alternative form of treatment. This study shows that compressed air therapy causes a cascade of morphometric and morphological changes in myofibres. Very little has been published on the morphological changes to skeletal muscle following massage of any form. A recent study by this group has shown that another form of massage using localised pressure, deep transverse friction (DTF) applied for 10 minutes to the untraumatised biceps femoris muscles of healthy rabbits also causes morphometric and ultrastructural changes in myofibres which persist for up to 6 days after treatment (Gregory et al 2003). There are however marked differences between the responses

Figure 12: 24 hours post-therapy - An extensive area of sarcomere disorganisation (SD) near the periphery of a fibre. Note the juxta-nuclear vacuolar and swollen SER (s).

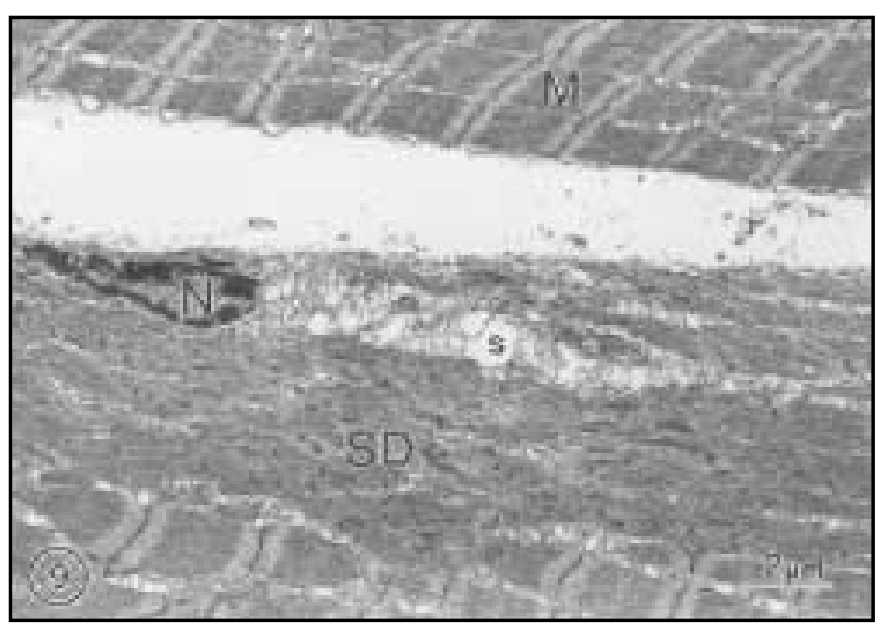

Figure 13: 24 hours post-therapy - Stretched sarcomeres (ss) and a contraction band (B) comprising 18 supercontracted sarcomeres. Note swollen SER (arrow ed) and crenated nucleus (N).

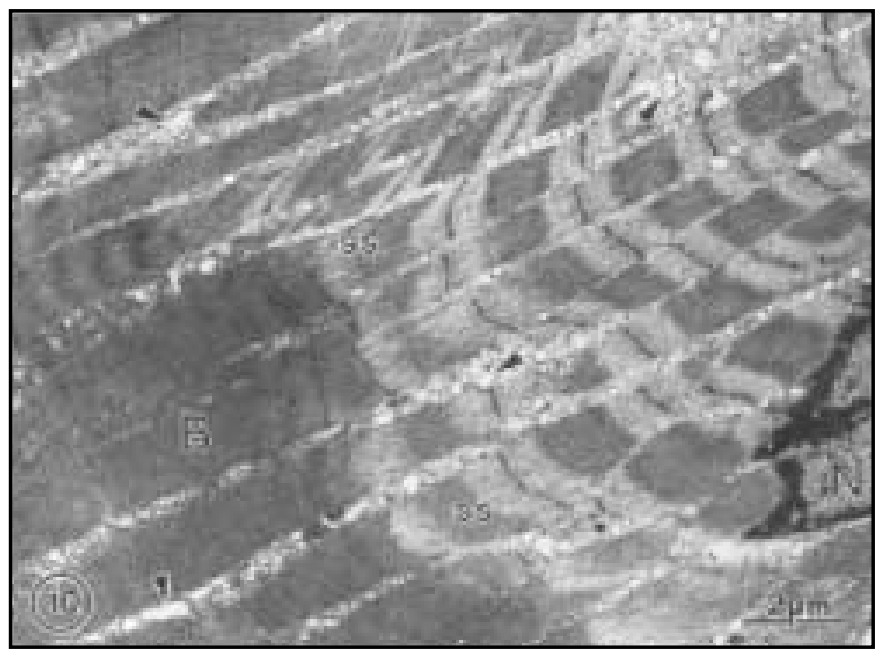

Figure 14: 6 days post-therapy - Detail of normal t-tubes (arrowed) and other sarcoplasmic organelles in a normal myofibre.

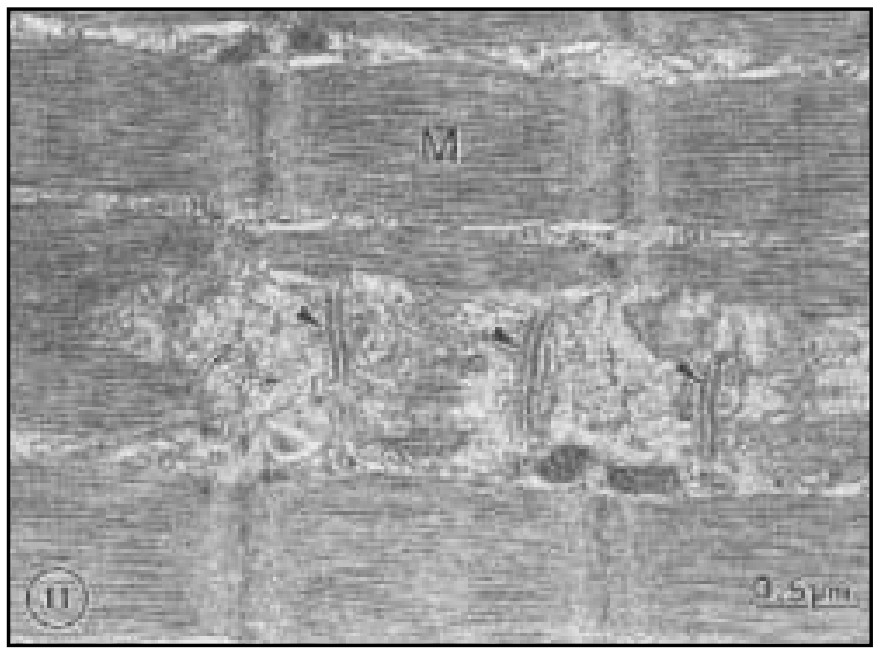

Figure 15: 6 days post-therapy - Swollen elements of the SER (s) in an otherwise normal myofibre.

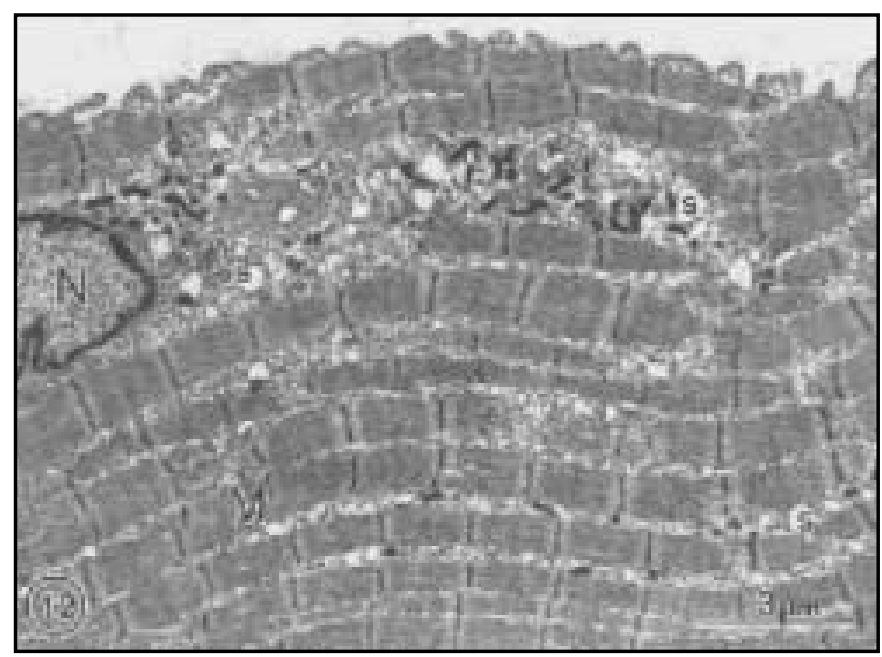


to the two treatment modalities, the main features of which will be described.

Compressed air therapy causes a significant increase in mean myofibre diameters of $17 \%(\mathrm{p}<0.001)$ within 10 minutes of treatment. Twenty-four hours later, mean fibre diameter was still increased by $5.5 \%(\mathrm{p}<0.001)$ and six days later, treated myofibres were on average $5 \%$ smaller than those in control muscle ( $\mathrm{p}<0.05)$. With DTF, myofibre diameters had increased $13.5 \%$ immediately after treatment and were maximally enlarged 24 hours after treatment $18 \%$ ( $p<0.001)$. By 6 days they had returned to normal diameter.

Following compressed air massage there was asynchrony of Z-bands across a number of fibres in each specimen. This appeared to be due to an apparent lengthening or "stretching" of some sarcomeres, especially those near the sarcolemma on both sides or on one side of a fibre. Associated with this were subsarcolemmal spaces filled with glycogen, mitochondria or small sarcoplasmic organelles. The SR in most fibres was swollen and in many cases vacuoles filled with fluid, appeared to have "budded" from the cisternae. While the SR was dilated, the t-tubes appeared normal.

In contrast, supercontraction bands stretching the full breadth of many fibres was the most conspicuous pathophysiological change immediately following DTF. No supercontraction was noted immediately after compressed air therapy. Supercontraction was associated with the displacement of mitochondria and other sarcoplasmic organelles from their normal positions between myofibrils, to subsarcolemmal and juxta-nuclear regions, with little morphological evidence of myofibre or organelle oedema.

Supercontraction has been reported as a common feature of muscle injury (Reddy et al 1991) and is thought to be caused by a massive increase in sarcoplasmic $\mathrm{Ca}^{++}$concentration. High sarcoplasmic $\mathrm{Ca}^{++}$is thought to be a consequence of impaired mitochondria failing to provide enough energy to power the $\mathrm{Ca}^{++}$ion pumps that transfer $\mathrm{Ca}^{++}$ions from the sarcoplasm to the sarcoplasmic reticulum (Hawke and Garry 2001). There may also be calcium influx through injury to the sarcolemma. The large supercontracted areas in many fibres suggest that immediately following DTF, myofibre contractile function may be impaired.

Other alterations seen immediately after compressed air therapy but not after DTF were the large, sometimes fluid filled intermyofibrillar spaces, occasional swollen mitochondria and swollen elements of the sarcoplasmic reticulum. Although sarcolemmae were intact, and appeared morphologically normal, the increase of fluid in the sarcosol indicated that the membrane had become permeable to extracellular fluids. These observations support the morphometric data and the increase in size of myofibres after compressed air treatment is probably as a consequence of local oedema. The swollen subsarcolemmal mitochondria are suggestive of functional impairment and failure to provide ATP to power the sarcolemmal and sarcoplasmic reticula ion pumps may explain the oedema.

Although there was still evidence of myofibre oedema and swelling and vacuolation of the SR, 24 hours after compressed air massage this was reduced. Occasional internalised nuclei were observed in some fibres. However, the most notable and regularly occurring phenomenon was areas of disruption of sarcomere and myofibril integrity. In such areas "fragments" of Z-band material were dispersed within a disorganised morass of myofilaments. In some fibres, sarcomere disorganisation was restricted to 3 or 4 sarcomeres in a single myofibril while in others, the disorganisation extended over a significant proportion of the fibre. The presence of internalised nuclei and myofibrillar disorganisation have been reported as evidence of both myofibre degeneration and regeneration (Hawke and Garry 2001).

Twenty-four hours after DTF, supercontraction was not noted and myofibres were characterised by an oedematous increase in the space between myofibrils. Two types of inclusions were noted. Type 1 inclusions occurred in large numbers and presented as amorphous, "colloidal" deposits within the rough sarcoplasmic reticulum while type 2 presented as groups of membranous whorls, or myelin figures in the intermyofibrillar spaces. The latter were probably the remnants of mitochondria damaged by DTF while the former resemble the sacs of hydrolytic enzymes described by (Gregory et al 1982) in pathological cardiac muscle. No inclusions of any type were observed in any myofibre treated with compressed air.

Six days after compressed air treatment, most fibres appeared morphologically normal with normal mitochondria, nuclei and other non-reticular, sarcoplasmic organelles. Occasional fibres had dilated sarcoplasmic reticulum and intermyofibrillar vacuoles were still present with normal mitochondria. Fibre size was reduced. This may be an indication of completion of repair of a sublethal injury. DTF treated fibres were still oedematous, retained occasional type 1 and 2 inclusions, internalised nuclei and disorganised aggregates of myofilaments were present in some fibres. The presence of internalised nuclei suggests that the repair process in the DTF treated animals was still underway 6 days after treatment.

To summarise, DTF initially causes supercontraction of fibres with mild oedema whereas compressed air therapy causes only myofibre oedema. Twenty four hours after DTF, the muscle becomes most oedematous and contains numerous inclusion bodies suggestive of significant injury, while after compressed air treatment oedema is receding and there is evidence of myofibre repair. Six days after DTF, myofibres still show pathological features together with phenomena associated with myofibre repair while cells exposed to compressed air therapy have normal ultrastructure. The morphological changes noted suggest that DTF causes a more severe injury to untraumatised rabbit skeletal muscle than compressed air therapy.

A possible explanation for this is that the applied pressure is localised to one area in DTF whereas in compressed air therapy, the pressure is applied over a wider area. This effectively reduces the "pressure dose" at any given site. The pressures transferred to subcutaneous tissue and muscle have not been reported for DTF. Compressed air therapy at 1 Bar through a $5 \mathrm{~mm}$ outlet hole, produces an average pressure in muscle of $60.7 \pm 21.4 \mathrm{mmHg}$ with the highest pressure recorded being $84 \mathrm{mmHg}$ (Mars 2002). In addition, compressed air therapy 
has been shown to significantly reduce the temperature of skin and subcutaneous tissues (Mars 2003). Cooling is known to slow metabolism and it is possible that the reduced pressure dose and the reduction in temperature during the compressed air massage are responsible for the difference in the initial and longer-term response to the two types of treatment.

\section{CONCLUSION}

The results of this study show that 10 minutes of compressed air therapy at 1 Bar causes a cascade of reversible morphometric and morphological changes in untraumatised rabbit skeletal muscle fibres. Myofibres become oedematous shortly after compressed air therapy with separation of myofibrils and swelling of some mitochondria and elements of the smooth endoplasmic reticulum. Twenty four hours after treatment, myofibre oedema is reduced and there is evidence of myofibril reorganisation and regeneration. Six days after massage, most myofibres appear normal. The difference in the morphological characteristics of reversible injury of myofibres after DTF and compressed air massage is ascribed to the more diffuse pressure applied to the tissues and the cooling effect that compressed air treatment has on both skin and muscle during the massage procedure. Clinical studies evaluating the use of compressed air therapy as an alternative form of pressure massage are required.

\section{REFERENCES}

Beard G, Wood EC 1965 History of Massage. In Massage manipulation and traction, Ed Licht S. Waverly Press, Baltimore

Cady SH, Jones GF 1997 Massage therapy as a workplace intervention for reduction of stress. Perceptual and Motor Skills 84:157-158

Casley-Smith J, Boris M, Wendorf S, Lasinski B 1998 Treatment of lymphedema of the arm - the Casley-Smith Method: A non-invasive method produces continued reduction. Cancer Supplement 83: 2843-2860

Deane MN, Gregory MA, Mars M 2002 Histological and morphometric changes in untraumatised skeletal muscle treated with deep transverse friction. South African Journal of Physiotherapy 58:28-35

Diego MA, Field T, Hernandez-Reif M, Shaw KH, Friedman L, Ironson G $2001 \mathrm{HIV}$ adolescents show improvement in immune function following massage therapy. International Journal of Neurosciences 106:265-274

Dubowitz V 1985 Normal Muscle. In: Dubowitz V, Sewry C Fitzsimons RB (ed) Muscle biopsy a practical approach, pp 82-128. Bailliere Tindall, London

Fiaschi F, Francesconi G, Fiumicelli SNA, Camici M 1998 Manual lymphatic drainage for chronic post-mastectomy lymphodema treatment. Panminerva Medica 40:48-50

Field T, Henteleff T, Hernandez-Reif M, Martinez E, Mavunda K, Kuhn C, Schanberg S 1998 Children with asthma have improved pulmonary function after massage therapy. Journal of Pediatrics $132: 854-858$
Field T, Hernandez-Reif M, Seligman S, Krasnegor J, Sunshine W, Rivas-Chacon R, Schanberg S, Kuhn C 1997a Juvenile rheumatoid arthritis: Benefits from massage therapy. Journal of Pediatric Psychology 22:607-617

Field T, Hernandez-Reif M, Shaw KH, LaGreca A, Schanberg S, Kuhn C 1997b Glucose levels decreased after giving massage therapy to children with Diabetes Mellitus. Diabetes Spectryn 10:23-25

Field TM 1998 Masage therapy effects. American Physiologist 53:1270-1281

Fritschy D, de Gautard R 1988 Jumper's knee and ultrasonography. American Journal of Sports Medicine 16:637-640

Gimblett PA, Saville J, Ebrall P 1999 A conservative management protocol for calcific tendinitis of the shoulder. Journal of Manipulative and Physiological Therapeutics 22:622-627

Goats GC 1994 The scientific basis of an ancient art: Part 2. Physiological and therapeutic effects. British Journal of Sports Medicine 28:153-156

Gregory MA, Deane MN, Mars M 2003 Ultrastructural changes in untraumatised rabbit skeletal muscle treated with deep transverse friction. Physiotherapy 89:408-416

Gregory MA, Mars M, Deane MN 2002 The effect of deep transverse friction on the morphology of untraumatised rabbit skeletal muscle. Proceedings International Conference of Electron Microscopy 15: 321-322

Gregory MA, Olmesdahl PJ, Whitton ID 1982 An ultrastructural study of the origin and function of basophilic degeneration in human cardiac muscle - cardiac colloid type 1. Journal of Pathology 138:337-351

Hammer WI 1993 The use of transverse friction massage in the management of chronic bursitis of the hip or shoulder. Journal of Manipulative and Physiological Therapeutics 16:107-111

Hawke TJ Garry DJ 2001 Myogenic satellite cells: physiology to molecular biology. Journal of Applied Physiology 91:534-551

Hunter G 1994 Specific soft tissue mobilisations in the treatment of soft tissue lesions. Physiotherapy 80:15- 21

Ironson G, Field T, Scafidi F, Kumar M, Patarca R, Price A, Goncalves A, Hashimoto M, Kumar A, Burman I, Tetenman C, Fletcher MA 1996 Massage therapy is associated with enhancement of the immune system's cytotoxic capacity. International Journal of Neurosciences 84:205-218

Kaments HL 1960 History of Massage. In Massage manipulation and traction, Ed Licht S. Waverly Press, Baltimore

Karnovsky MJ 1965 A formaldehyde glutaraldehyde fixative of high osmolarity for use in electron microscopy. Journal of Cell Biology 27:127-139

Kurz W, Kurz R, Litmanovitch YI, Romanoff II, Pfeiffer Y, Sulman FG 1981 Effect of manual lymphdrainage massage on blood components and urinary neurohormones in chronic lymphhedema. Angiology $32: 119-127$ 
Mars M 2002 Jet therapy - its effect on skin blood flow, muscle pressure and blood parameters. South African Journal of Surgery 40:23

Mars M 2003 Compressed air massage, a new treatment modality? Medicine and Science in Sports and Exercise 35:S92

Mars M, Gregory MA 1991 A histometric analysis of skeletal myofibers following $90 \mathrm{~min}$ of tourniquet ischemia and reperfusion. Journal of Surgical Research 50:191-195

Mars M, Hadley GP 1998 Raised intracompartmental pressure and compartment syndromes. Injury 29:403-411

Norris C 1993 Sports Injuries pp109-111. Butterworth Heinemann, New York

Olmesdahl PJ, Gregory MA, Cameron EWJ 1979 Ultrastructural artefacts in biopsied normal myocardial biopsy in man. Thorax 34:82-90

Reddy A, Reedy M, Best T 1991 Evaluation of strain injuries in rabbit skeletal muscle using a single fibre model. Surgery Forum 12:44
Reynolds ES 1963 The use of lead citrate at high $\mathrm{pH}$ as an electron opaque stain in electron microscopy. Journal of Cell Biology 17: 208-210

Smith LL, Keating MN, Holbert D, Spratt DJ, McCammon MR, Smith SS, Israel RG 1994 The effects of athletic massage on delayed onset muscle soreness, creatine kinase and neutrophil count: A preliminary report . JOSPT: The journal of orthopaedic and sports physical therapy 19:93-99

Spurr A 1969 A low viscosity epoxy resin embedding medium for electron microscopy. Journal of Ultrastructure Research 26:31-43

Sunshine W, Field T, Quintino O, Fierro K, Kuhn C, Burman I, Schanberg S 1996 Fibromyalgia benefits from massage therapy and transcutaneous electrical stimulation. Journal of Clinical Rheumatology $2: 18-22$

Waza U, Katakotha Y, Sagara Y 1993 Anaesthesia and Analgesia 76:783-785

MEDIA RELEASE

\section{Positive Clinical Trial Results Imminent}

$\mathbf{J}^{\mathrm{e}}$ et Therapy International opened its new head office and 'benchmark' clinic in Durban North recently. At the opening, Garth O'Connor who started Jet Therapy in 1999 expressed his "gratitude to the medical team who has assisted and guided me in getting these trials done."

"I know people, especially the medical fraternity, were sceptical when we started," O'Connor admits, "but the recent clinical trial results are hugely positive and I am thrilled that the scientific community is now providing real proof about the bona fides of Jet Therapy."

Over the past year Prof Maurice Mars, from the Nelson Mandela School of Medicine, University of KwaZulu-Natal in Durban, Professor Mike Gregory of the University of KwaZulu-Natal and Chief Surgeon at R K Khan Hospital in Chatsworth, Mr Desai, have put Jet Therapy through a process of rigorous clinical evaluations and scientific trials and now have a better understanding of how and why circulation therapy, using pure compressed air, does what it claims to do.

The medical team have shown that the massage increases blood flow through the skin, that the process dilates micro-blood vessels and that the therapy causes less reversible muscle tissue damage than does conventional crossfriction massage. In addition, the clinical studies have shown that the massage cools muscle tissue to the same extent as an external ice pack, while at the same time reducing pain.

The results of a comprehensive clinical trial currently being done at the Chatsworth Hospital, where diabetic patients with severe foot ulcers, under threat of leg amputations are being treated by a team of doctors under the guidance of Mr. Desai, have not yet been published. However, all the preliminary results show a significant reduction in healing times which could play a major role in reducing leg amputations, which appears to vindicate some of the spectacular claims O'Connor originally made.

Going forward, rapid development in techniques for cellulite reduction, running parallel with the numerous medical trials across a range of circulatory conditions, will probably mean a split in the current retail business between cosmetic "circulation stations" and medical treatment points at a clinic level.

Jet Therapy International currently operates in South Africa, and other parts of Africa such as Botswana, Namibia, Zimbabwe and Mauritius and in Australia, USA Greece, Cyprus and the United Kingdom. This month the group opened new clinics in Swaziland, Scotland and Holland.

For more information on Jet Therapy, call: (031) 563-6949 


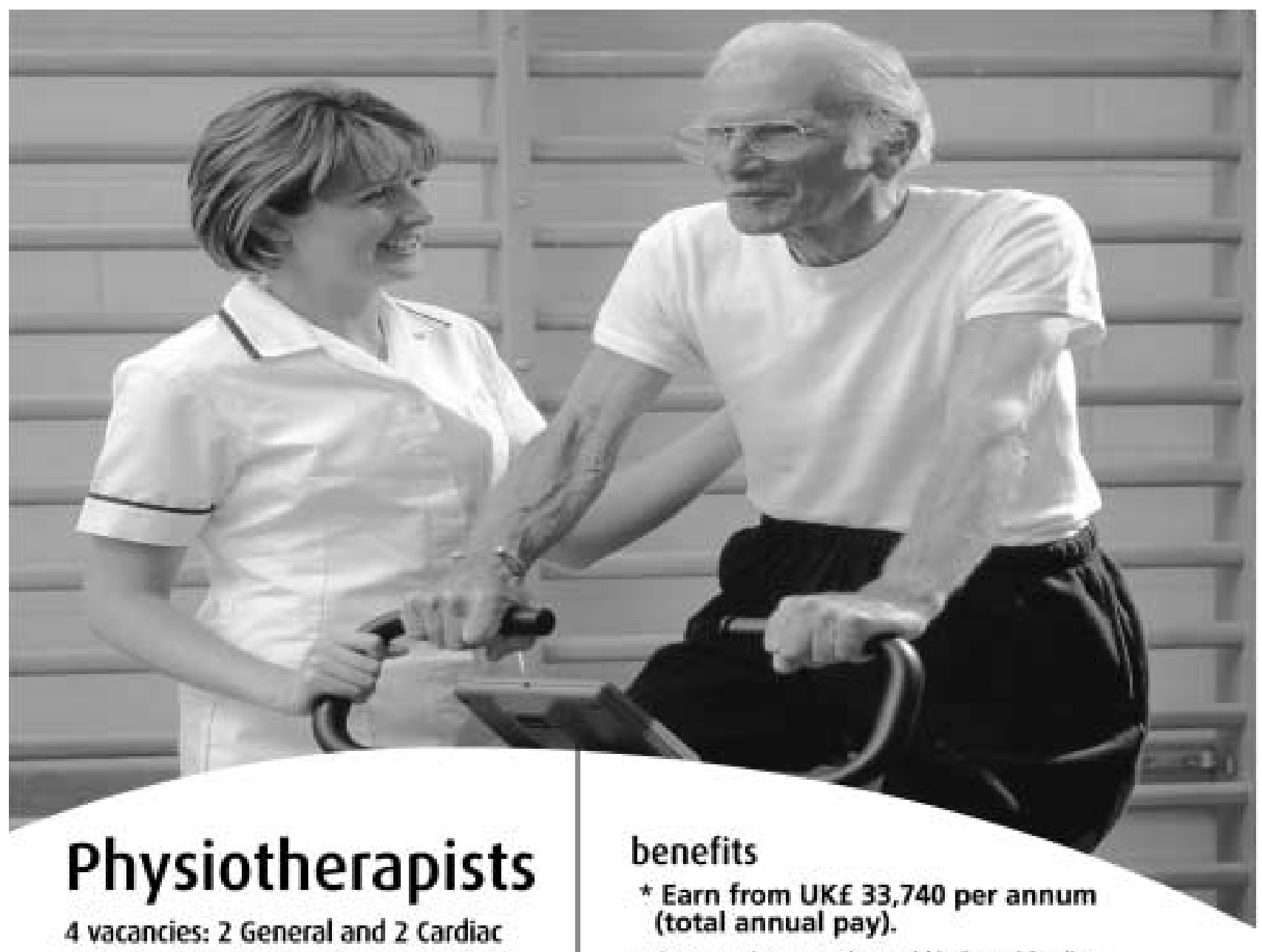
with Saudi Arabia's premier employer...

Saudi Aramco, the world's leading petroleum enterprise, has an outstanding reputation for providing comprehensive medical care to its employees and their dependents. The Company healthcare facilities are superbly equipped and are staffed by over 4,000 medical professionals. Facilities include a 480 -bed hospital in Dhahran and an 80-bed hospital in Al Hasa.

\section{Job Description}

Successful candidates will have a Bachelor's degree in Physical Therapy, a current licence to practice and at least two years post-qualification experience, treating a wide range of medical conditions. Both inpatient and outpatient services are provided with treatments ranging from manual therapy to running advanced diagnostic equipment. The Cardiac Physical Therapist should also be familiar with cardiac rehabilitation in a inpatient and outpatient setting.

- An attractive nett salary paid in Pound Sterling (average monthly take home from UKC 2,500 or $\left.\mathrm{R} 29,000^{*}\right)$

- An open-ended contract with high savings potential

- Paid vacation entitlement of 30 days each calendar year

- Quality single status housing in company communities

- Free medical care in Saudi Arabia

- Personal effects shipment and excess baggage allowances

- Access to some of the finest social and recreational facilities in the Middle East

- Paid airfares allowing full flexibility with holiday travel.

* The Rand salary estimate is based on current exchange rates. As the payroll is Sterling, the Aand equivalent may be higher or lower than the amount quoted due to changes in exchange rates.

Please send your CV to Samantha du Toit at: Latimer Recruitment (Pty) Ltd, PO Box 309,

Constantia 7848.

Tel: (021) 7021322

Fax: (021) 7021311

Email: samanthablatimer.co.za

Web; www.latimercoza 